\title{
SALUD FÍSICA DESDE EL MODELO DE CREENCIAS EN ESTUDIANTES DE LA UNIVERSIDAD DE PAMPLONA.
}

\author{
Mantilla, S. ${ }^{1}$ Mogollon, C. ${ }^{2}$ Villamizar, C. ${ }^{3}$
}

1. Toloza Mantilla Sonia. (2015) Fisioterapeuta (Universidad Industrial de Santander), Especialista en Pedagogía Universitaria (Universidad de Pamplona), Magíster en Salud Pública (Universidad Nacional de Colombia), Doctora en Ejercicio físico, Fisioterapia y Salud (Universidad de Murcia).

Docente Asistente Facultad de Salud, Universidad de Pamplona (Colombia). sonia.mantilla@unipamplona.edu.co

2. Mogollon Canal Olga. (2015). Psicóloga (Universidad Santo Tomás), Magíster en Educación (Universidad Autónoma de Bucaramanga UNAB- Instituto Tecnológico de Monterrey ITESM), Doctora en Líneas Actuales de Investigación en Psicología (Universidad Pública de Navarra).

Docente Asociada, Facultad de Salud, Universidad de Pamplona (Colombia).

olgamariela@unipamplona.edu.co

3. Villamizar Carmen. (2015). Licenciada en Matemáticas y Computación (Universidad de Pamplona), Especialista en Educación Matemática (Universidad de Pamplona), Magíster en Educación Matemática (Universidad Nacional Experimental del Táchira).

Docente Facultad de Ciencias Básicas, Universidad de Pamplona (Colombia).

cevill@unipamplona.edu.co

\section{RESUMEN}

Objetivo: Describir las relaciones entre género y facultad en aspectos de la salud física de un grupo de estudiantes de la Universidad de Pamplona, desde el modelo de creencias durante el primer período académico de 2013.

Metodología: Estudio descriptivo de tipo trasversal con una muestra de 836 estudiantes escogidos por muestreo aleatorio simple a los cuales se les aplicó cuestionario para evaluar diferentes aspectos de la salud física de los universitarios, a partir del modelo de creencias. En el análisis estadístico se emplearon correspondencias múltiples.

Resultados: Severidad percibida: Se encontró un alto consumo de comida chatarra. Las mujeres consideran que su estado de salud física actual es poco satisfactorio, tienen problemas de visión y el estrés es un factor que afecta el estado físico.

Susceptibilidad percibida: Contrario a los hombres, la mayor parte de mujeres considera que algunas veces presentan dificultades para mantener su salud física. La mayoría de estudiantes permanecen sentados entre 1 y 10 horas. La frecuencia de consumo diario de comidas oscila entre dos y cinco.

Beneficios percibidos: Alta frecuencia de repuesta positiva frente a la posibilidad de practicarse una cirugía si tuviera una enfermedad, seguir las recomendaciones después de una consulta 
médica, caminar de manera regular y preocupación de los seres más cercanos por la salud física. Bajo consumo de frutas y verduras.

Barreras percibidas: La mitad de los estudiantes considera que es más fácil encontrar excusas para no hacer ejercicio que para salir a hacerlo.

Motivación para la salud: Algunas mujeres practican baloncesto, natación y realizan actividad física en el tiempo libre para disminuir problemas de salud. La mayoría de los hombres realizan actividades para disminuir problemas de salud, practican tenis y fútbol, en el tiempo libre realizan deportes y otras actividades físicas.

Claves para la acción: La actividad física predilecta en las mujeres es la ejecución de aeróbicos, mientras que los hombres prefieren montar en bicicleta, caminar y en menor medida hacer aeróbicos. Contrario a las mujeres, los hombres se ejercitan junto con su familia o amigos. La mayor parte de los universitarios realiza un esfuerzo por consumir alimentos que favorecen su digestión, evita consumir alimentos altos en grasa, sal o azúcar.

Conclusiones: Se identificaron comportamientos que podrían considerarse como amenazas para la salud de los universitarios, pero también otros, que podrían ofrecer posibilidades para disminuirlas. Se requiere generar estrategias en el contexto universitario que ofrezcan oportunidades saludables y faciliten la adopción de hábitos a favor de la salud en los universitarios.

Palabras clave: actitudes y prácticas en salud física, estudiantes universitarios.

\section{ABSTRACT}

Purpose: The principal objective of this research was to describe the relationship between gender and College career, in aspects of physical health, of a group of students from the University of Pamplona, from the belief model during the first semester of 2013.

Methodology: A descriptive study of cross type with a sample of 836 students selected by simple random sampling, which was applied questionnaire to assess different aspects of physical health of college, from belief model. The statistical analysis used multiple correspondence.

Results: Perceived Severity: high consumption of junk food was found. Women consider your current physical health is unsatisfactory, have vision problems and stress is a factor affecting fitness.

Perceived susceptibility: Unlike men, most women think that sometimes have difficulty maintaining their physical health. Most students remain seated between 1 and 10 hours. The frequency of daily consumption of meals varies between two and five.

Perceived benefits: High frequency of positive response to the possibility of have surgery if I had a disease, follow the recommendations after a medical appointment, walk regularly and concern of the closest physical health beings. Low fruit and vegetables.

Perceived barriers: Half of the students considered it easy to find excuses not to exercise it to go to.

Motivation for health: Some women play basketball, swim and perform physical activity in leisure time to reduce health problems. Most men perform activities to reduce health problems, play tennis and football, leisure time perform sports and other physical activities.

Keys to action: favorite physical activity in women is running aerobics, while men prefer bicycling, walking and less aerobic. Unlike women, men are exercising together with family or friends. Most college makes an effort to consume foods that promote digestion, avoid eating foods high in fat, salt or sugar. 
Conclusions: The research identified behaviors that could be considered as threats to the health of the College students, but also others that could offer possibilities to reduce them were identified. There is a need to generate strategies in the university context that provide healthy opportunities and facilitate the adoption of habits for college students.

Key words: attitudes and practices in physical health, college students.

\section{INTRODUCCIÓN}

El conjunto de hábitos cotidianos de comportamiento determinan el estilo de vida de las personas. Un estilo de vida, se ha definido como el patrón de conducta individual que demuestra cierta consistencia en el tiempo, bajo condiciones más o menos constantes y que puede constituirse en dimensiones de riesgo 0 de seguridad dependiendo de su naturaleza (Roth E., 1990).

Aunque sería difícil encontrar conductas humanas que no tuvieran alguna relación con la salud, existe un grupo de patrones que componen los factores de riesgo más importantes de los principales problemas de salud en la vida actual, tales como el sedentarismo, las relaciones sexuales inseguras, el desequilibrio nutricional, el consumo de alcohol, el tabaquismo, el consumo de sustancias psicoactivas, la poca o nula participación en programas de promoción de la salud, el incumplimiento de las recomendaciones médicas y el uso inadecuado de los servicios de salud, entre otros.

Por el contrario, los estilos de vida saludables son comportamientos de salud, creencias, conocimientos, hábitos $y$ acciones de las personas para mantener, restablecer o mejorar su salud, que surgen de la interacción entre ellas y su entorno. Se refieren a formas cotidianas de vivir que aplican para las diferentes esferas del campo vital de una persona, con el fin de satisfacer adecuadamente sus necesidades y a su vez optimizar su calidad de vida.

Según el modelo de creencias, éstos factores que determinan las conductas de salud pueden ser de dos tipos: los que hacen referencia a la percepción de amenazas sobre la propia salud y los que incluyen creencias de los individuos sobre la posibilidad de reducir esas amenazas. La percepción de amenazas sobre la propia salud se encuentra determinada por los valores generales sobre la salud, las creencias específicas sobre la propia vulnerabilidad ante la enfermedad (susceptibilidad percibida) y las creencias sobre la gravedad de la enfermedad (severidad percibida). Por otra parte, las creencias sobre la posibilidad de reducir la amenaza se encuentran en función de la creencia en la eficacia de las medidas concretas para reducir las amenazas y la convicción de que los beneficios de la medida superan los costos (beneficios y barreras percibidas). Por último, la presencia de estímulos internos o externos que podrían considerarse como fuentes de motivación y claves para la acción (Yeung y cols., 2004).

La interacción entre las amenazas y la posibilidad de reducirlas, puede complementar el diseño y aplicación de modelos adecuados para la disminución 0 prevención de factores de riesgo en diversos grupos poblacionales que produzcan el impacto deseado o por lo menos aumenten las probabilidades de éxito.

Los adultos jóvenes forman un grupo especialmente vulnerable frente al impacto que ocasiona la relación de factores determinantes de estilos de vida, siendo muchos de ellos estudiantes universitarios. Ellos se caracterizan por formar un 
segmento poblacional fácilmente identificable y accesible, que ocupan una posición significativa en la vida pública y el cambio social, no solo en el presente sino también en el futuro (Steptoe A., 2001), son estratégicamente importantes en la promoción de patrones de comportamiento saludables y prevención de factores de riesgo.

Para muchos jóvenes y adolescentes, el rol universitario puede ser excitante o más aún, estresante (Dinger M., 1997). Tradicionalmente, los jóvenes ingresan en la universidad cerca de los 18 años, enfrentándose a los cambios que llevan consigo la vida académica, los nuevos amigos y el ambiente que les rodea. Junto con estos cambios y nuevas responsabilidades, los universitarios tienen mayor libertad y mayor control sobre sus estilos de vida. Sin embargo, diversas investigaciones (Steptoe A., 2002; Dinger M., 1997; Mantilla C., 2011) han demostrado que, en general, los universitarios adoptan hábitos que representan riesgos para su salud, incluyendo el tabaquismo, consumo de alcohol, inactividad física, prácticas alimentarias no saludables, relaciones sexuales inseguras, consumo de estupefacientes, entre otros; todos estos comportamientos inciden en su calidad de vida.

A partir de estas premisas, se ha desarrollado el presente trabajo, con el propósito de describir las relaciones entre género y facultad en aspectos de la salud física de un grupo de estudiantes de la Universidad de Pamplona, desde el modelo de creencias, con el fin de identificar conductas de riesgo o amenazas para la salud, que puedan disminuir o prevenirse.

\section{MÉTODO}

Esta investigación descriptiva, de tipo transversal hace parte del macroproyecto "Niveles de Salud de profesores, estudiantes y administrativos de la Universidad de Pamplona, desde el modelo de creencias", perteneciente al Grupo de Investigación Psicología Y Sociedad. Esta investigación está radicada en la Vicerrectoría de Investigaciones de la Universidad de Pamplona dentro de la convocatoria permanente. Se realizó con 836 estudiantes universitarios de la Universidad de Pamplona, los cuales fueron seleccionados a través de muestreo aleatorio simple a partir de una población de 8360 estudiantes matriculados en el primer semestre académico del año 2013, pertenecientes a la sede central de la Universidad de Pamplona.

La recolección de datos se realizó durante el primer semestre de 2013, previo diligenciamiento de consentimiento informado por los participantes y bajo la supervisión y aprobación del Comité de ética e impacto ambiental de la Universidad de Pamplona. Se aplicó un cuestionario diseñado para evaluar diferentes aspectos de la salud física de los universitarios, el cual cuenta con 6 subcategorías establecidas desde el modelo de creencias, referente teórico de la psicología de la salud (Moreno E., 2003) susceptibilidad percibida, severidad percibida, beneficios percibidos, barreras percibidas, motivaciones para la salud y claves para la acción. Además se incluyeron preguntas referentes a información sociodemográfica. Los instrumentos fueron validados a partir de dos fuentes: validación por jueces, para lo cual se seleccionaron expertos en el campo de psicología clínica y de la salud y de salud física, quienes evaluaron los instrumentos, teniendo en cuenta la pertinencia, suficiencia y redacción de cada uno de los puntos; y prueba piloto a partir de las recomendaciones de los jueces, aplicada a una muestra de 27 sujetos de una institución de educación superior diferente a la Universidad de Pamplona.

Para la recolección de la información el equipo investigador contó con el apoyo de estudiantes de práctica del programa de psicología, quienes previa capacitación y 
entrenamiento colaboraron con la aplicación de algunos instrumentos.

Procesamiento y análisis de la información: Para el procesamiento y análisis de la información, los datos fueron consignados en un formato de ingreso, de acuerdo con las variables del estudio y la fuente de la información. El análisis estadístico se realizó con técnicas estadísticas multivariantes, específicamente el análisis de correspondencias múltiples. Teniendo como eje de variables "género" y "facultad", se establecieron las características de cada uno de los comportamientos de salud física establecidos dentro de las categorías del modelo de creencias.

\section{RESULTADOS}

Del total de la muestra de 836 sujetos, 353 estudiantes eran mujeres $(42,2 \%)$ y 483 eran hombres $(57,8 \%)$. Respecto a la edad, se encontró una media de 20,94 años, con un valor mínimo de 16 años y máximo 39 años, con desviación típica de 2,88 años. La distribución de la muestra de acuerdo a las facultades fue: 347 estudiantes (41,5\%) pertenecían a la Facultad de Salud, 196 estudiantes $(23,4 \%)$ pertenecían a la Facultad de Ingenierías y Arquitectura, 92 estudiantes $(11 \%)$ pertenecian a la Facultad de Artes y Humanidades, 90 estudiantes $(10,8 \%)$ a la Facultad de Ciencias Económicas, 53 estudiantes $(6,3 \%)$ a la Facultad de Educación, 33 estudiantes $(3,9 \%)$ a la Facultad de Ciencias Agrarias y 25 estudiantes (3\%) a la Facultad de Ciencias Básicas.

La información referente a cada componente del modelo de creencias por género $y$ facultad se describe a continuación:

Suceptibidad percibida:

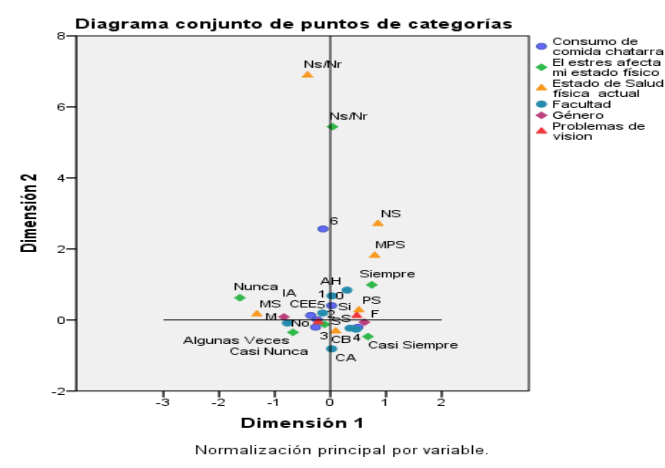

La mayor parte de mujeres de las facultades de Salud, Educación, Artes y Humanidades, Ciencias Agrarias, Ciencias Económicas y Empresariales considera de manera aceptable, que tiene energía física para realizar actividades. Expresan que el número de comidas al día oscila entre 1-2 y 2-3; mientras que el número de horas que permanecen sentadas oscila entre 5-10, 11$15,16-20$, y algunas veces presentan dificultades para mantener la salud física.

El género masculino de las facultades de Ingenierías y Arquitectura y Ciencias Básicas indican que tienen excelente energía física para realizar actividades. El número de comidas diarias oscila entre 3 a 4 ó 4 a 5 o algunos indican que 5 a 6 comidas al día; el número de horas que permanecen sentados está entre 6 a 10 y casi nunca o nunca tienen dificultades para mantener la salud física.

Severidad percibida:

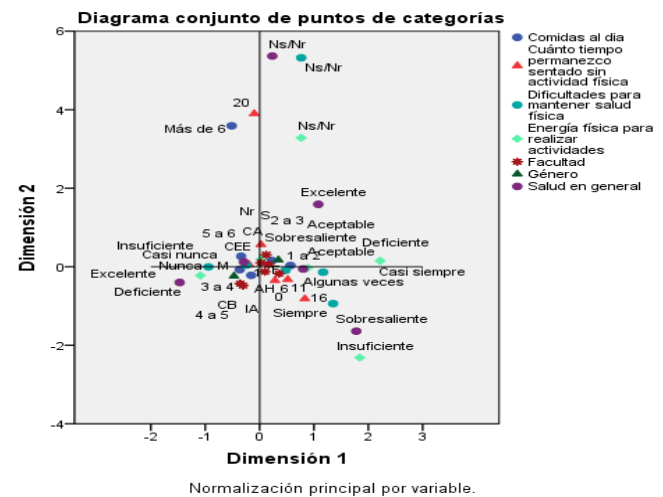


Las personas de género femenino de las facultades de Ciencias Básicas, Ciencias Agrarias y salud consideran que casi siempre el estrés afecta su estado físico, una vez al mes consumen comida chatarra, y el estado de salud física actual es poco satisfactorio.

Otro grupo de género femenino de las facultades de Artes y Humanidades y Educación considera que el estrés siempre afecta su estado físico, su estado de salud es poco 0 nada satisfactorio y tienen problemas de visión. En cuanto al consumo de comida chatarra, algunas indican que lo hacen al menos una vez por día y otras nunca consumen.

Los estudiantes de sexo masculino, principalmente de las facultades de Ciencias Económicas y empresariales e Ingenierías y Arquitectura consideran que pocas veces el estrés afecta su estado físico, indican que su estado de salud actual es muy satisfactorio, no tienen problemas de visión. Cada dos o tres días consumen comida chatarra.

Beneficios percibidos:

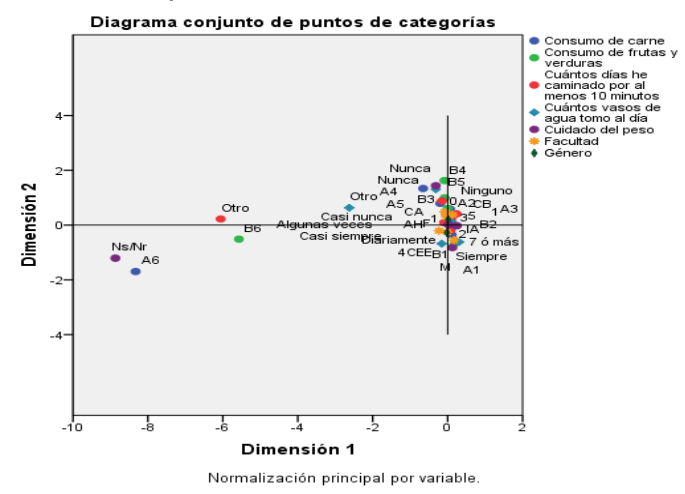

En éste componente no se apreciaron diferencias marcadas entre género $y$ facultad. En general, el $85 \%$ de los estudiantes respondió que si se practicaría una cirugía si tuviera una enfermedad, frente a un $9 \%$ que no lo haría. El $88 \%$ de los estudiantes consideran que siempre 0 casi siempre que se enferman, sus seres más cercanos se preocupan por su salud
Física, respecto a un $10 \%$ que considera que casi nunca es así.

El $61,2 \%$ de los estudiantes considera que los remedios caseros son más efectivos que ir al médico, frente a $30 \%$ que creen que no es así. Sin embargo, cuando se pregunta a los universitarios si después de una consulta médica, sigue las recomendaciones que le dan, el $70 \%$ manifiesta que si sigue las recomendaciones que le dan, mientras que el $22 \%$ de los universitarios algunas veces lo hace y el $7 \%$ casi nunca. $76 \%$ de los estudiantes nunca ha asistido al nutricionista, el $20 \%$ algunas veces y sólo el $3 \%$ si ha acudido alguna vez.

Frente a la práctica de actividad física de baja intensidad, el $60,6 \%$ de los universitarios manifiesta que camina todos los días, $18,8 \%$ de los universitarios caminan entre tres a seis días, el $14,8 \%$ de los universitarios camina uno a tres días, mientras que el $5 \%$ manifiesta que no camina.

Respecto al consumo de agua, el $43 \%$ de los estudiantes manifiestan que consumen 1 o dos vasos de agua diarios, el $26,2 \%$ de estudiantes consume de 2 a 4 vasos, el $15,7 \%$ de universitarios consume de cuatro a seis vasos y el $7 \%$ nunca beben agua.

Respecto al consumo de carne, el $53,9 \%$ de los universitarios encuestados consumen algún tipo de carne al menos una vez por día, el $32,7 \%$ de los universitarios consumen algún tipo de carne cada dos 0 tres días, mientras que el $9 \%$ de los estudiantes lo hacen sólo una vez por semana.

En cuanto al consumo de frutas y verduras, el $37,7 \%$ de los estudiantes consumen frutas y/o verduras al menos una vez por día, el 32,9\% de universitarios consumen frutas y/o verduras cada dos o tres días, mientras que el $20 \%$ sólo lo hace una vez por semana. 
Frente al cuidado del peso corporal para estar en buen estado de salud física, el $47 \%$ de los estudiantes actualmente cuida de su peso para estar en buen estado de salud física, el $27,8 \%$ de los estudiantes, algunas veces lo hace y el $24 \%$ no cuidan su peso corporal para estar en buen estado de salud física. Por otra parte, el $53 \%$ de universitarios creen que el contexto en donde se desenvuelven les permite mantener una adecuada salud física, $34,4 \%$ creen que algunas veces es así y $12 \%$ no.

Barreras percibidas:

En éste componente no se apreciaron diferencias marcadas entre género $y$ facultad.

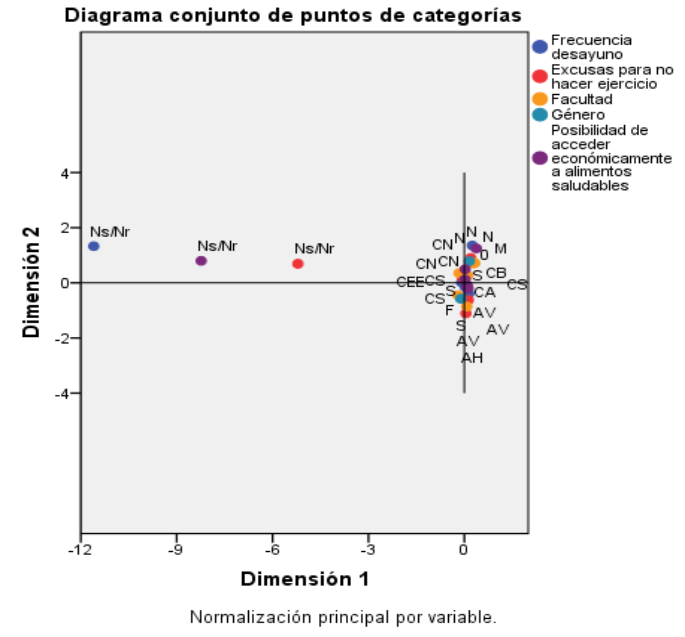

Cuando se pregunta a los universitarios si es más fácil encontrar excusas para NO hacer ejercicio que para salir a hacerlo?, la mitad de los estudiantes considera que es más fácil encontrar excusas para NO hacer ejercicio que para salir a hacerlo, frente a la otra mitad que considera lo contrario.

Respecto a la frecuencia con la que los estudiantes desayunan, el $49 \%$ de universitarios manifiesta que casi siempre desayuna, $25,8 \%$ de estudiantes algunas veces desayuna y $20 \%$ de estudiantes nunca lo hace.
El $57 \%$ de los estudiantes manifiesta que siempre 0 casi siempre tiene la posibilidad de acceder económicamente a alimentos saludables, $33 \%$ de los estudiantes manifiesta que sólo algunas veces tiene esa posibilidad, mientras que el $8,5 \%$ no tiene la posibilidad de acceder económicamente a alimentos saludables.

Motivación para la salud

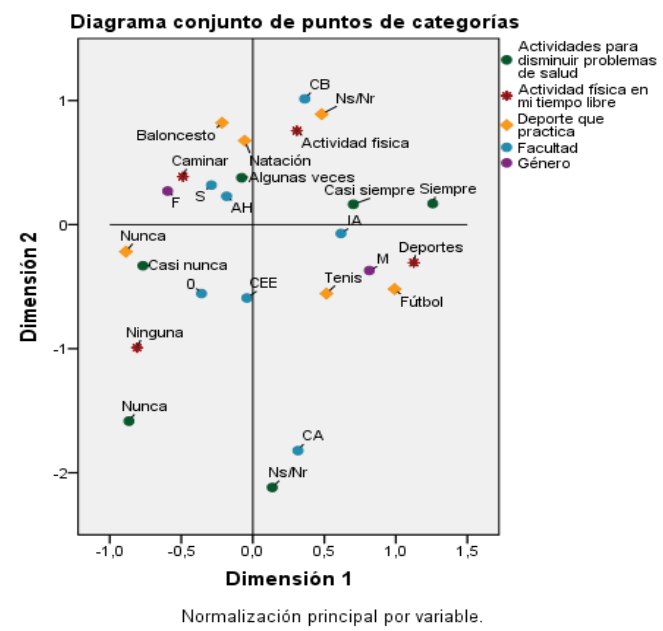

En esta dimensión el género femenino de las facultades de Salud y Artes y Humanidades practican baloncesto 0 natación, algunas veces realizan actividad física en el tiempo libre, principalmente caminar para disminuir problemas de salud. Mientras que quienes pertenecen a las facultades de Educación y Ciencias Económicas y Empresariales, casi nunca realizan actividades para disminuir problemas de salud, nunca practican deportes y tampoco realizan actividad física en el tiempo libre.

Los estudiantes de género masculino de la facultad de Ciencias Agrarias no saben o no responden a si realizan actividades para disminuir problemas de salud, mientras que Ingenierías y Arquitectura y en menor grado Ciencias Básicas indican que siempre o casi siempre lo hacen. Los deportes que los hombres practican en su tiempo libre son tenis y fútbol $u$ otras actividades físicas. 
Claves para la acción:

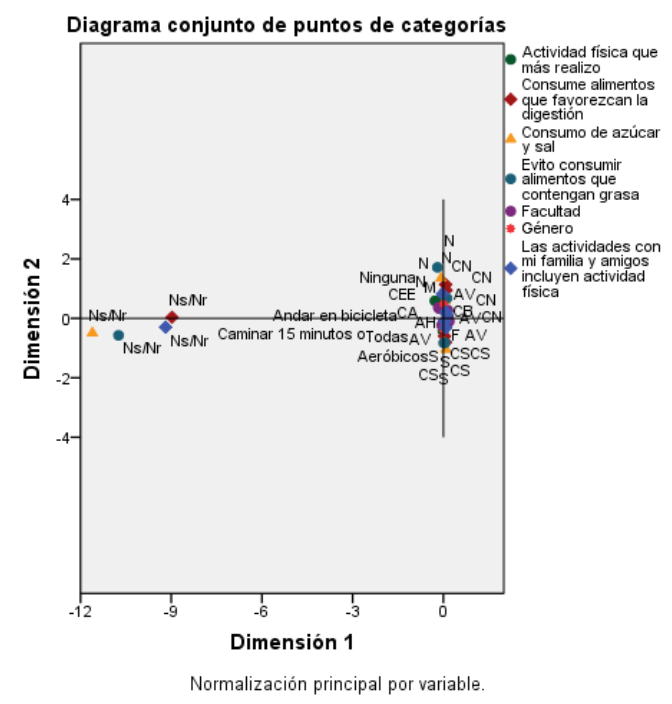

Las mujeres de las facultades de Salud, Educación y Artes y Humanidades prefieren realizar aeróbicos y en las actividades con la familia y amigos casi nunca se incluye la actividad física. Mientras que los hombres de las facultades de Ingenierías y Arquitectura, Ciencias Económicas y Empresariales, Ciencias Agrarias y Ciencias Básicas, prefieren como actividad física montar en bicicleta, caminar 15 minutos 0 hacer aeróbicos y en las actividades con la familia o amigos se involucra la actividad física.

En cuanto al consumo de azúcar, sal y grasas no se aprecian diferencias entre los grupos por género y facultad. Al respecto, la mayor parte de los universitarios evita consumir alimentos altos en azúcar, grasas y/o sal, mientras que otros casi nunca 0 nunca los evitan.

\section{DISCUSIÓN}

Se ha caracterizado la salud física de un grupo de estudiantes de la Universidad de Pamplona, desde el modelo de creencias. En general, los resultados del estudio identifican amenazas para la salud, pero también posibilidades para disminuirlas.

En el componente de severidad percibida se encontró que en ambos géneros existe un consumo frecuente de comida chatarra. En las mujeres, el estrés es un factor que afecta el estado físico. Ellas además consideran que su estado de salud física actual es poco satisfactorio y tienen problemas de visión. No ocurre lo mismo con los hombres, para quienes el estrés pocas veces afecta su estado físico, su estado de salud actual es muy satisfactorio y no tienen problemas de visión. La baja percepción del estado de salud en las mujeres podría sustentarse en las apreciaciones de Denton y otros (2004), quienes afirmaron que la salud de las mujeres está más influenciada por determinantes estructurales y psicosociales como el excesivo autocontrol, estrés y baja autoestima.

A partir de los hallazgos en la dimensión de severidad percibida, se requiere diseñar e implementar acciones para el control, disminución y prevención del estrés, así como también para promover entornos armónicos y equilibrados, promover una alimentación saludable y disminuir el consumo de comida chatarra. Además, generar acciones de mantenimiento para favorecer la perspectiva de salud percibida, pero sobre todo para modificar la percepción de aquellos quienes consideran que su salud actual es poco satisfactoria.

En lo referente a su percepción sobre el riesgo de enfermar (susceptibilidad percibida) (Moreno E., 2003), la mayor parte de mujeres considera que algunas veces presentan dificultades para mantener su salud física, aun así manifiestan que tienen energía para realizar actividades. Por su parte, los hombres manifiestan que casi nunca 0 nunca tienen dificultades para mantener su salud física, tienen una excelente energía física para realizar actividades. Otros aspectos evaluados se relacionan con hábitos de los estudiantes que podrían considerarse como un riesgo real de enfermar. Estos aspectos incluyen el tiempo que permanece sentado en un día, a partir del cual se determina el sedentarismo 
o la ausencia de actividad física; el número de comidas que consume diariamente y si considera que se alimenta de manera adecuada. Respecto al tiempo de sedentarismo diario el cual se evaluó preguntando por el tiempo de permanencia sedente en un día, se encontró que la mayoría de estudiantes permanecen sentados entre 1 y 10 horas. Llama la atención que algunos reportaron tiempos de permanencia en sentado entre 11 y 24 horas, puesto que la pregunta no incluía los tiempos dedicados al descanso nocturno. Se identifica un tiempo considerable dedicado a actividades sedentarias, el cual puede influir en la percepción de la salud, energía y rendimiento académico de los estudiantes. Al respecto se requiere generar estrategias que incentiven en los estudiantes la disminución de hábitos que favorezcan el sedentarismo y por el contrario, se promuevan comportamientos hacia la adopción y mantenimiento de la práctica de actividad física.

Respecto a los hábitos alimentarios que podrían llegar a ser riesgo de enfermedad, la mayoría de estudiantes considera que actualmente su alimentación es adecuada, mientras que sólo el $13 \%$ manifiesta que no es así. La frecuencia de consumo diario de comidas oscila entre dos y cinco. Sin embargo, llama la atención que algunos estudiantes manifiestan que consumen sólo una a dos comidas diarias $(14,5 \%)$ y otros entre 5 y 7 comidas diarias (6\%). Puede considerarse que una parte de los universitarios además de que perciben que actualmente su alimentación no es adecuada, consumen sólo una a dos comidas diarias; situación que influye en la percepción de su salud, energía, rendimiento académico y en la ocurrencia de enfermedad. Por lo cual, además de generar estrategias que incentiven en los estudiantes la alimentación adecuada, se requiere de un monitoreo de los lugares que dispensan la alimentación a los universitarios para favorecer que realmente se ofrezcan alimentos saludables.
En la dimensión de beneficios percibidos se incluyen situaciones que en algún momento los universitarios pudieran considerar como ciertamente efectivas a la hora de enfrentarse con la enfermedad (Moreno E., 2003). Entre esas situaciones se encontró un alto porcentaje de repuesta positiva frente a la posibilidad de practicarse una cirugía si tuviera una enfermedad, seguir las recomendaciones después de una consulta médica, caminar de manera regular e interés y preocupación de los seres más cercanos por su salud física. En éste último aspecto, una parte de los universitarios encuestados percibe que cuando se enferman, sus seres más cercanos casi nunca se preocupan por su salud, lo cual podría suceder debido a que las familias de una buena parte de los estudiantes viven en otros lugares distantes, lo cual dificulta la atención en caso de enfermar. Al preguntar a los universitarios si considera que los remedios caseros son más efectivos que ir al médico, la mayoría de los estudiantes considera que los remedios caseros son más efectivos que ir al médico. Esta situación podría generar una reflexión frente a la efectividad y credibilidad de las prácticas de intervención médica actuales, pero además de las creencias de la efectividad de los remedios caseros en la enfermedad. Frente a la práctica de actividad física de baja intensidad, aunque la mayor parte de los universitarios manifiesta que camina de manera regular lo cual influye de manera positiva sobre el mantenimiento de la salud, otra parte de universitarios no lo hace; por lo cual se requiere generar estrategias para la promoción del mantenimiento del hábito de caminar más de diez minutos diariamente y además para promover la adopción de éste hábito.

Aunque el consumo de frutas y verduras es considerado como un beneficio percibido por representar uno de los principales factores protectores frente a la ocurrencia de enfermedad (Atalah E., 2000; Uauy R., 
2006) se ha identificado que para los universitarios participantes en éste estudio es bajo; lo cual es consistente con otros estudios realizados en el contexto latinoamericano (Troncoso C., 2011). Además, la mitad de universitarios creen que el contexto en donde se desenvuelven les permite mantener una adecuada salud física, otros creen que sólo algunas veces es así y muy pocos consideran que no. Al respecto, se requiere generar estrategias a nivel institucional y municipal que ofrezcan oportunidades saludables y faciliten la adopción de hábitos a favor de la salud en los universitarios.

Respecto a las barreras percibidas 0 situaciones que podrían influenciar de manera negativa la adopción 0 mantenimiento de comportamientos saludables (Moreno E.,, 2003) no se encontraron diferencias entre hombres y mujeres. Sin embargo, la mitad de los estudiantes considera que es más fácil encontrar excusas para NO hacer ejercicio que para salir a hacerlo, frente a la otra mitad que considera lo contrario. Otros autores también han encontrado ésta barrera, que además se considera como la principal razón para el abandono de las actividades deportivas (Kimm Y., 2006).

Respecto a la frecuencia con la que los estudiantes desayunan, el $49 \%$ de universitarios manifiesta que casi siempre desayuna, $25,8 \%$ de estudiantes algunas veces desayuna y $20 \%$ de estudiantes nunca lo hace. Teniendo en cuenta que el desayuno es un hábito que protector de la salud, pues a partir de éste se obtienen los requerimientos energéticos para iniciar la rutina diaria, se requiere que no sólo un alto porcentaje de los estudiantes lo hayan asumido como un hábito, sino que la totalidad de estudiantes lo adopte y lo mantenga en el tiempo. Aunque la mayoría de estudiantes tiene la posibilidad de acceder económicamente a alimentos saludables, en un poco porcentaje se incluyen estudiantes que no tienen ésta posibilidad.

Dentro de las situaciones que motivan o influyen de manera positiva en la adopción de comportamientos para la salud(Moreno E., 2003) identificadas en este estudio, se encontró que mientras algunas mujeres, principalmente de las facultades de Salud y Artes y Humanidades practican baloncesto o natación, algunas veces realizan actividad física en el tiempo libre para disminuir problemas de salud y otras casi nunca realizan actividades para disminuir problemas de salud, nunca practican deportes y tampoco realizan actividad física en el tiempo libre. Esto resulta preocupante teniendo en cuenta que la práctica de actividad física regular es un factor que incide de manera positiva en el rendimiento académico y en la protección de la salud, mientras que la inactividad física es un factor de riesgo para la adquisición de ciertas enfermedades. (American College of sports medicine, 2010). Por su parte, la mayoría de los hombres realizan actividades para disminuir problemas de salud, practican tenis y fútbol, en el tiempo libre realizan deportes y otras actividades físicas.

Las diferencias en la práctica de actividad física entre hombres y mujeres registradas en el presente estudio hacen parte de un patrón constante, ya que los hombres registran mayores niveles de actividad física que las mujeres (Varela-Mato, Cancela, Ayan, Martín, Molina, 2012; Talaei y otros 2013; Mantilla S., Gómez A., Hidalgo M., 2011)). Se ha reportado que éstas diferencias se deben a la preferencia por ciertas actividades (Buckworth y Nigg, 2004), a la diferencia de roles entre género (Cabrera, Gomez, Mateus, 2004) y a la influencia de factores sociales y culturales que determinan esos roles (Häkkinen, Järvelin, Rosenquist, Laitinen, 2006; Denton, Prus, Walters, 2004).

Las actividades que pueden considerarse como estímulos desencadenantes del 
proceso de toma de decisiones frente a la salud o claves para la acción (Moreno E., 2003) en el presente estudio hacen referencia a las actividades físicas que más realizan los universitarios en la vida cotidiana, a la inclusión de actividad física en su vida social, al esfuerzo por consumir alimentos que favorecen su digestión y el bajo consumo se sal, azúcares y alimentos con alto contenido de grasas. Se ha identificado que la actividad física predilecta en las mujeres es la ejecución de aeróbicos, mientras que los hombres prefieren montar en bicicleta, caminar y en menor medida hacer aeróbicos. Respecto a la inclusión de actividad física en la vida social, los hombres se ejercitan junto con su familia 0 amigos, mientras que en las mujeres no sucede lo mismo. La mayor parte de los universitarios realiza un esfuerzo por consumir alimentos que favorecen su digestión, evita consumir alimentos altos en grasa, sal o azúcar mientras que otros casi nunca o nunca lo evitan.

Los resultados de éste estudio aportan a la construcción de un diagnóstico institucional que se constituye como una herramienta de orientación y apoyo para facilitar la labor de las autoridades universitarias, bienestar universitario y comunidad académica, en los procesos de planificación y diseño de intervenciones que tengan el objetivo de capacitar y organizar la vida saludable en la Universidad, a partir de referentes nacionales e internacionales relacionados con el tema, favoreciendo la retención estudiantil.

\section{CONCLUSIONES}

Los resultados del presente estudio aportan el conocimiento de los hábitos de vida de los universitarios, puesto que permiten identificar comportamientos que podrían considerarse como amenazas para la salud, pero también otros, que podrían ofrecer posibilidades para disminuirlas. Se requiere generar estrategias en el contexto universitario que ofrezcan oportunidades saludables y faciliten la adopción de hábitos a favor de la salud en los universitarios.

Dichas estrategias deben proyectarse a nivel interdisciplinario, puesto que la salud es un compuesto de varias áreas, como se ha podido establecer en el marco teórico de este macroproyecto. Las políticas de Bienestar Universitario se ampliarán así al trabajo conjunto de todas las áreas $y$ departamentos de la Facultad de Salud, siendo el estudiante el beneficiado en todo su proyecto de vida saludable, lo cual redundará en su rendimiento académico y su futuro desempeño profesional.

\section{REFERENCIAS BIBLIOGRÁFICAS}

Atalah E., Urteaga C., Rebolledo A., Medina E., Csendes A. (2000). Factores de riesgo del cáncer de mama en mujeres de Santiago. Revista médica de Chile, 128(2), 137-143.

American College of sports medicine. (2010). ACSM guidelines for exercise testing and prescription. Walter Thompson editor. Lippincott Williams and Wilkins.

Buckworth J., Nigg C. (2004). Physical activity, exercise and sedentary behaviour in college students. Journal of American College Health. 53: 28-34.

Cabrera G., Gomez L., Mateus J. (2004). Leisure time physical activity among women in a neighbourhood in Bogotá, Colombia: prevalence and socio- demographic correlates. Revista de Salud Pública. 20:1103-1109.

Denton M., Prus S., Walters V. (2004). Gender differences in health: a canadian study of the psychosocial, structural and behavioural determinants of health. Social Science and Medicine. 58:2585-2600.

Dinger M,, Waigandt A. (1997). Dietary intake and physical activity behaviours of 
male and female collage students. American Journal of Health promotion. 11:360-362.

Häkkinen U., Järvelin M., Rosenquist G., Laitinen J. (2006). Health, schooling and lifestyle among young adults in Finland. Health Economics. 15:1201-1216.

Kimm Y., Glynn W., McMahon P., Voorhees C., Striegel-Moore, H., Daniels R. (2006). Self-perceived barriers to activity participation among sedentary adolescent girls. Medicine and Science in Sports and Exercise, 38(3), 534-540.

Mantilla S., Gómez A., Hidalgo M. (2011) Actividad física, tabaquismo y consumo de alcohol, en un grupo de estudiantes universitarios Revista de Salud Pública. 13 (5), 748-758. Universidad Nacional de Colombia, Bogotá, Colombia.

Moreno E., Gil J. (2003) El Modelo de Creencias de Salud: Revisión Teórica,Consideración Crítica y Propuesta Alternativa. I: Hacia un Análisis Funcional de las Creencias en Salud International Journal of Psychology and Psychological Therapy. 3(1), 91-109.

Steptoe A., Wardle J. (2001) Health behaviour, risk awareness and emotional well-being in students from eastern Europe and western Europe. Social Science and Medicine. 53:1621-1630.

Steptoe A., Phil D., Cui W., Bellisle F., Zotti A., Baranyai R. (2002) Trends in smoking, diet, physical exercise and attitudes toward health in european university students from
13 countries, 1990-2000. Preventive Medicine. 35:97-104.

Serra Puyal, J.R.; Generelo Lanaspa, E. y Zaragoza Casterad, J. (2010). Barreras para la realización de actividad física en adolescentes en la provincia de Huesca. Revista Internacional de Medicina y Ciencias de la Actividad Física y el Deporte vol. 10 (39) pp. 470-482.

Talaei M., Rabiei K., Talaei Z., Amiri N., Zolfaghari B., Kabiri P., Sarrafzadegan N. (2013). Physical activity, sex, and socioeconomic status: A population based study. ARYA Atherosclerosys. 9(1):51-60.

Troncoso C. (2011). Percepción de la alimentación durante la etapa de formación universitaria, Chile. Rev Costarr Salud Pública 2011; 20: 83-89.

Uauy R. (2006). Importancia de las frutas y verduras en la prevención de enfermedades crónicas. Revista chilena de nutrición, 33(Supl. 1), 224-225.

Varela-Mato V., Cancela J., Ayan C., Martín V., Molina A (2012). Lifestyle and health among Spanish university students: differences by gender and academic discipline. Int J Environ Res Public Health. 9(8):2728-2741.

Yeung A., Chang D., Gresham R jr., Nierenberg A., Fava M. (2004). "Illness Beliefs of Depressed Chinese American Patients in Primary Care". The Journal of Nervous and Mental Disease. 192(4):324327. 